\title{
Bloch waves and non-propagating modes in photonic crystals
}

\author{
R. Smaâli, D. Felbacq, G. Granet \\ LASMEA UMR-CNRS 6602 \\ Complexe des Cézeaux \\ 63177 Aubière Cedex \\ France
}

(November 9, 2018)

\begin{abstract}
We investigate the propagation of electromagnetic waves in finite photonic band gap structures. We analyze the phenomenon of conduction and forbidden bands and we show that two regimes are to be distinguished with respect to the existence of a strong field near the interfaces. We precise the domain for which an effective medium theory is sounded.
\end{abstract}

\section{INTRODUCTION}

The theoretical and numerical study of photonic band gap materials (see [1] for an exhaustive bibliography) may be led both from the point of view of the spectrum, with the computation of the band structure by using Bloch wave theory and the related quantities (Density of States and so on), or from the point of view of the scattering theory [2]. In case of a scattering experiment, which of course involves a finite structure, Bloch waves are not a priori sufficient to describe the electromagnetic field because of the existence of evanescent and anti-evanescent waves, linked to the boundary of the device. Of course these non-propagating modes are of prime importance in the band gaps, as they represent entirely the total field inside the photonic crystal, but they may also induce strong effects for frequencies lying inside the conduction bands.

In the context of electromagnetic optics and the spectacular effects that can be obtained using photonic crystals (the so-called ultra-refraction effects [3, 4.,5,6.7.87]) a theoretical approach only involving equivalent medium theories, group velocity [3, [4,5] and more generally quantities only derived from the band structure is certainly incomplete. Previous studies have shown the importance of considering the isoenergy diagram and not only the dispersion diagram [8,9]. In the present work, our aim is to analyse the relative importance of the 
evanescent waves (the near-field) for describing the electromagnetic field inside a photonic crystal. More precisely, we study the ratios of the projection of the field on the propagating and non-propagating modes, and we precise the conditions under which Bloch waves can describe entirely the scattering behaviour of a photonic crystal.

\section{PROPAGATING AND NON-PROPAGATING MODES}

The photonic crystal under study is made of a stack of gratings. For the numerical applications we use square dielectric rods in a dielectric matrix (fig. 1). The basic layer of the device is made of one grating, consisting of a slab of rods between two homogeneous slabs above and below (fig.1): it covers a band $\mathbb{R} \times[-h / 2, h / 2]$. The period of the grating is $d$. We use time-harmonic fields with a time dependence of $e^{-i \omega t}$ and $k=\frac{2 \pi}{\lambda}$ denotes the wavenumber in vacuum. We have chosen this particular geometry (square rods and rectangular symmetry) and polarization for the sake of simplicity (both on the numerical level and for the theoretical exposition). However the theoretical framework is quite general and works as well for more complicated situations (see [10] for an exposition of the transfer matrix method for 3D obstacles).

Our aim is to characterize the field inside the photonic crystal. Due to the translational invariance of the problem, we look for pseudo-periodic fields in the $x$ direction: $u(x+d, y)=\exp (i \alpha d) u(x, y)$, where $\alpha$ belongs to the interval $Y=[-\pi / d, \pi / d[$. In a diffraction problem, where the grating is illuminated by a plane wave under the incidence $\theta$ (fig. 1), we have $\alpha \equiv k \sin \theta \bmod (\pi / d)$. From grating theory [11], we can define the trans-

fer matrix of the basic layer, which is the operator $\widetilde{\mathbf{T}}_{(\alpha, \lambda)} \operatorname{linking}\left(u(x, h / 2), \partial_{y} u(x, h / 2)\right)$ to $\left(u(x,-h / 2), \partial_{y} u(x,-h / 2)\right)$ (in ordinary differential equations theory this is the monodromy matrix). There are many very good numerical methods for computing the field diffracted by a grating and hence the transfer matrix, even for stacks of gratings [12,13, 14].

On the upper (resp. lower) side of a basic cell, we expand the field on a Rayleigh basis:

$$
u(x, h / 2)=\sum_{n}\left(A_{n}^{+}+A_{n}^{-}\right) e^{i \alpha_{n} x}, u(x,-h / 2)=\sum_{n}\left(B_{n}^{+}+B_{n}^{-}\right) e^{i \alpha_{n} x} .
$$

where $\alpha_{n}=\alpha+n K, K=\frac{2 \pi}{d}$. The values of the normal derivatives $\left.\partial_{y} u\right|_{y= \pm h / 2}$ write:

$$
\partial_{y} u(x, h / 2)=\sum_{n} i \beta_{n}\left(A_{n}^{+}-A_{n}^{-}\right) e^{i \alpha_{n} x}, \partial_{y} u(x,-h / 2)=\sum_{n} i \beta_{n}\left(B_{n}^{+}-B_{n}^{-}\right) e^{i \alpha_{n} x} .
$$

The knowledge of $\widehat{A^{ \pm}}=\left\{A_{n}^{ \pm}\right\}_{n}$ (resp. $\widehat{B^{ \pm}}=\left\{B_{n}^{ \pm}\right\}$) gives the value of the derivatives. Therefore, rather than computing the monodromy matrix as defined above, we compute the matrix $\mathbf{T}_{(\alpha, \lambda)}$ such that

$$
\mathbf{T}_{(\alpha, \lambda)}\left(\begin{array}{c}
\widehat{A^{+}} \\
\widehat{A^{-}}
\end{array}\right)=\left(\begin{array}{c}
\widehat{B^{+}} \\
\widehat{B^{-}}
\end{array}\right)
$$

The point is to analyze the spectrum of $\mathbf{T}$. For symmetry reasons, the spectrum $s p(\mathbf{T})$ of $\mathbf{T}$ is invariant under $\tau \rightarrow \tau^{-1}$ (this is easily seen in case of a lamellar grating, but the proof is slightly more involved in case of a $y$-dependent medium), then we 
can distinguish between eigenvalues of modulus 1 that are necessarily finitely many, and eigenvalues that do not belong to the unit circle of the complex plane. Let us denote $\left.\left.e^{i \beta h}, \beta \in\right]-\frac{\pi}{h}, \frac{\pi}{h}\right]$, an eigenvalue of $\mathbf{T}_{(\alpha, \lambda)}$ of modulus one, and $\psi$ an associated eigenvector then we have $\mathbf{T}\left(\widehat{\psi^{+}}, \widehat{\psi^{-}}\right)=e^{i \beta h}\left(\widehat{\psi^{+}}, \widehat{\psi^{-}}\right)$or else: $\psi(x, y+h)=e^{i \beta h} \psi(x, y)$ this means that $(x, y) \rightarrow e^{i \alpha x} \psi(x, y)$ is a Bloch wave associated to the Bloch vector $(\alpha, \beta)$. That way we can easily compute the dispersion curves at a given wavenumber $k$. Moreover, we can also compute the non-propagating modes inside the crystal: they correspond to eigenvectors associated with eigenvalues that are not of modulus one. We have thus obtained a decomposition of the modes by means of a family of monodromy operators parametrized by $\alpha \in Y$. As it as been said before, from the scattering point of view, the parameter $\alpha$ is equal to $k \sin \theta$ so that we study the spectrum of $\mathbf{T}_{(\theta, \lambda)}=\mathbf{T}_{(k \sin \theta, \lambda)}$.

We can now give the following definitions.

Definition 1 We call relative gap an interval of wavelengths $I_{\theta}$, at a given incidence $\theta$, for which $\mathbf{T}_{(\theta, \lambda)}$ has no eigenvalues of modulus one and we call relative conduction band an interval $B_{\theta}$ of wavelengths where $\mathbf{T}_{(\theta, \lambda)}$ does have eigenvalues of modulus 1.

Definition $2 A$ total gap corresponds to the intersection of incident dependent gaps (and may be void).

\section{ANALYSIS OF THE SPECTRUM}

As it has already been stated, at a given wavelength, Bloch waves are not sufficient to compute the scattering properties of the crystal. In order to quantify the relative importance of the evanescent waves, we need to be able to get a decomposition of the field.

\section{A. Decomposition of the field}

Once the electromagnetic field is known on the upper face of the crystal (through the coefficients $\widehat{A^{ \pm}}$, obtained from a rigorous numerical method), it is possible to expand it on the various modes that exist in the grating layer. More precisely, except on a set of wavelengths of zero Lebesgue measure, matrix $\mathbf{T}_{(\alpha, \lambda)}$ can be put in diagonal form:

$$
\mathbf{T}_{(\alpha, \lambda)}=\mathbf{T}_{p} \oplus \mathbf{T}_{e} \oplus \mathbf{T}_{a}
$$

where $\mathbf{T}_{p}$ is a finite rank operator corresponding to propagative waves and $\mathbf{T}_{e}, \mathbf{T}_{a}$ correspond to the evanescent and anti-evanescent modes.

With this decomposition, the vector $\psi=\left(\widehat{\psi}^{+}, \widehat{\psi}^{-}\right)$writes $\psi=\psi_{p} \oplus \psi_{e} \oplus \psi_{a}$. Whence we define the branching ratios $\pi_{p}$ (resp. $\pi_{e}, \pi_{a}$ ) of the field on the propagating (resp. evanescent, anti-evanescent) modes by:

$$
\pi_{p}=\frac{\left\|\psi_{p}\right\|^{2}}{N(\psi)}, \pi_{e}=\frac{\left\|\psi_{e}\right\|^{2}}{N(\psi)}, \pi_{a}=\frac{\left\|\psi_{a}\right\|^{2}}{N(\psi)}
$$


where $N(\psi)=\left\|\psi_{p}\right\|^{2}+\left\|\psi_{e}\right\|^{2}+\left\|\psi_{a}\right\|^{2}$.

The point of the above decomposition is to quantify the relative importance of the various modes in the total field existing in the crystal, in order to understand to what extend the field is not solely described by Bloch waves.

\section{B. Cut wavelengths and classification of the conduction bands}

Let us now turn to some numerical computations. The relative permittivity of the rods is $\varepsilon_{2}=9$ and $\varepsilon_{1}=\varepsilon_{\text {ext }}=1$, the geometric parameters are $h=2.8, d=2.8, h_{1}=1.9, d_{1}=1$. The structure is made of one basic layer and we choose $\theta=30^{\circ}$ and $s$-polarized waves. In fig.2 (a), we give the absolute values of the eigenvalues of $\mathbf{T}_{\theta}(\lambda)$ versus the wavelength. The conduction bands are the regions with a horizontal straight line $(|\mu|=1)$. For each wavelength $\lambda$ there is a finite, possibly empty, set of eigenvalues of modulus one $\left\{e^{i \beta_{n}(\lambda)}\right\}_{n}$ and an infinite set of eigenvalues that do not belong to $\mathbb{U}=\{z \in \mathbb{C},|z|=1\}$ (in fig. 2 (b) we have plotted the real part of the spectrum of $\mathbf{T}_{(\theta, \lambda)}$, where the finite number of propagating modes may be observed). We have also plotted in fig.2 (c) the dispersion diagram $(\beta, \lambda / d)$. The comparison with fig. 2 (a) shows that the consideration of the complete spectrum of $\mathbf{T}_{(\theta, \lambda)}$, i.e. with the non-propagating modes, allows to understand that one should not treat on a different foot propagating and non-propagating modes because they are really the same physical entities, behaving differently according to the wavelength. When $\theta$ varies in ]$-\pi / 2, \pi / 2[$, the local gaps vary as shown in fig. 3 .

This first example is rather generic and shows that within a given conduction band, hence locally, it is possible to define continuous sections $\lambda \rightarrow \mu_{n}(\lambda) \in \operatorname{sp}\left(\mathbf{T}_{(\theta, \lambda)}\right)$ representing the evolution of the eigenvalues of the monodromy operator with respect to the wavelength. At some values of the wavelength however, these sections may encounter a bifurcation, or cutoff: the eigenvalue leaves $\mathbb{U}$ and the associated modes give rise to an evanescent mode and an anti-evanescent mode. At such a branch point, the section $\lambda \rightarrow \mu_{n}(\lambda)$ is not differentiable and may cross other sections. As a consequence, a global description of the sections is not possible in that case. This problem is quite a complicated one, for which there is a general theory [20,21], but even deriving a specific theory for our particular situation is quite a big task and beyond the scope of this work.

However, an easy simplification can be obtained by noting that the set of eigenvalues being invariant under $\tau \rightarrow \tau^{-1}$, it is natural to consider the quotient space $\operatorname{sp}\left(\mathbf{T}_{(\theta, \lambda)}\right) / \sim$ for the equivalence relation $\mu_{1} \sim \mu_{2}$ if $\mu_{1} \mu_{2}=1$, which amounts to identify two eigenvalues that are inverse one of the other. This operation gives a nice simplification but still does not allow to define global sections (a more detailed account of this situation will be given elsewhere [15]).

Remark 3 A very simple example of branch point is the extinction of a diffracted order in grating theory. Another elementary situation is that of a stratified medium (a Bragg mirror for instance) in which case there are only two propagative modes in the conduction bands and one evanescent and one anti-evanescent mode inside the gaps. A realization of the quotient space is obtained by considering $\left\{\frac{1}{2} \operatorname{tr}\left(\mathbf{T}_{(\theta, \lambda)}\right), \lambda \in \mathbb{R}^{+}\right\}$. In that case, this very set defines a global section and the quotient space $\operatorname{sp}\left(\mathbf{T}_{(\theta, \lambda)}\right) / \sim$ is a trivial fibred bundle. 
For a given incidence, a gap is then an interval of wavelengths over which all the propagative eigenvalues have encountered a bifurcation. In fig. 2 (a), we have this situation in the interval $(1.32,1.42)$.

When the wavelength tends to infinity, it is known that the device finally behaves as a homogeneous slab [16, [17, 18, 19], and then there are only two propagative modes (up and down), which means that all "sections" finally bifurcate definitely (see fig. 2 (a-b) for $\lambda / d>2.22$ ), except the one corresponding to the homogenization regime. In that case there are still evanescent (and anti-evanescent waves) but with a very huge damping exponent so that $\pi_{e}$ and $\pi_{a}$ are small.

However, before that regime, eigenvalues may experience a local bifurcation: that is they leave $\mathbb{U}$ over a finite interval but finally come back on it (in fig. 2 (b) this situation happens over the interval $(1.63,1.87))$. What is important to note is that such a local bifurcation may affect only one eigenvalue so that, whithin a conduction band there may be evanescent field coming from such a bifurcation, hence with a small damping exponent (this happens over the interval $(1.32,1.42)$ in fig. $2(a-b))$. This leads us to distinguish between both regimes and give the following definitions.

Definition $4 \mathrm{~A}$ conduction band is said local if among the evanescent modes within this band there is at least one mode corresponding to a local bifurcation. A conduction band is global if it is not local.

Let us now give some numerical examples of the various regimes described above. We give in fig.4 the absolute values of the eigenvalues of matrix $\mathbf{T}_{(0, \lambda)}$ (normal incidence) for a one layer structure with $\varepsilon_{\text {ext }}=2.26, \varepsilon_{1}=1, \varepsilon_{2}=4 h=1, d=1, d_{1}=0.5$ and the projection ratii $\pi_{p, e, a}$. The region $(1.28,1.37)$ corresponds to a local conduction band, i.e. in which there is a local bifurcation of an eigenvalue. We see that the part of the field on the nonpropagating modes is not at all negligible so that the field cannot be described solely by Bloch modes. On the contrary, for the interval $(1.72,1.8)$ the conduction band corresponds to a global bifurcation of two eigenvalues of modulus one and in that case the damping exponents are exponentially growing, so that almost Bloch waves only contribute to the description of the field.

A natural question is to know to what extend these situations persist when the number of layers is increased. We have computed the values of the branching ratios when the number $N$ of layers is $N=2,4,6$. The results are given in figures 5 (a-b-c). It can be seen that the branching ratios $\pi_{e}$ and $\pi_{p}$ exhibit an oscillatory behavior with respect to $\lambda$, which is probably linked to the excitation of resonances, but that the fraction of electromagnetic energy that is carried by the evanescent waves is not diminished. This means that a non negligible part of the field is localized near the interfaces, which can have substantial consequences on the propagation of a beam inside the structure (this situation will be analyzed in a forthcoming paper [17) but also on the local density of states for photons.

As an application, suppose now that the wavelength belongs to a global conduction band and is such that there is only one diffracted and one transmitted order; then from the knowledge of the transmission and reflection coefficients, it is possible to compute the $(2 \times 2)$ monodromy matrix of the device. In that case, we can obtain the superior envelope of the transmitted energy by considering only the transfer matrix of one basic layer [22]. We have 
shown in another article [23] that in a layer characterized by a $2 \times 2$ transfer matrix, the reflected and transmitted coefficients for $N$ layers can be obtained in close form :

$$
r_{N}(\lambda, \theta)=f \frac{\mu^{2 N}-1}{\mu^{2 N}-f g^{-1}}, t_{N}(\lambda, \theta)=\mu^{N} \frac{\left(1-f g^{-1}\right)}{\mu^{2 N}-f g^{-1}}
$$

where $\mu$ is an eigenvalue of $\mathbf{T}_{p}=\left(\mathbf{t}_{i j}\right)$ associated with an eigenvector $\mathbf{u}=\left(u_{1}, u_{2}\right)$, an eigenvector associated to $\mu^{-1}$ is denoted by $\mathbf{v}=\left(v_{1}, v_{2}\right)$ and, denoting $q\left(x_{1}, x_{2}\right)=\frac{i \beta_{0} x_{2}-x_{1}}{i \beta_{0} x_{2}+x_{1}}$, $\beta_{0}=\frac{2 \pi}{\lambda} \cos (\theta)$, functions $f$ and $g$ are defined by

$$
\begin{aligned}
& \text { if }(k, \theta) \in \mathbf{G} g(k, \theta)=q(\mathbf{v}), f(k, \theta)=q(\mathbf{w}) \\
& \text { if }(k, \theta) \in \mathbf{B}\left\{\begin{array}{l}
g(k, \theta)=q(\mathbf{v}), f(k, \theta)=q(\mathbf{w}) \text { if }|q(\mathbf{v})|<|q(\mathbf{w})| \\
g(k, \theta)=q(\mathbf{w}), f(k, \theta)=q(\mathbf{v}) \text { if }|q(\mathbf{w})|<|q(\mathbf{v})|
\end{array}\right.
\end{aligned}
$$

where $\mathbf{G}=\left\{(k, \theta),\left|\operatorname{tr}\left(\mathbf{T}_{p}\right)\right|>2\right\}$ and $\mathbf{B}=\left\{(k, \theta),\left|\operatorname{tr}\left(\mathbf{T}_{p}\right)\right|<2\right\}$.

The superior envelope $R_{\infty}$ of the reflected energy, and conversely the inferior envelope of the transmitted energy $T_{\infty}$ are given by [22]:

$$
\begin{aligned}
T_{\infty} & =\frac{4-\operatorname{tr}\left(\mathbf{T}_{p}\right)^{2}}{\left(\mathbf{t}_{12} \beta_{0}-\mathbf{t}_{21} \beta_{0}^{-1}\right)^{2}} \\
R_{\infty} & =1-T_{\infty}
\end{aligned}
$$

A direct application of these formulas show a very accurate result in fig.6 (a-b) for a global conduction band.

We can conclude by the following:

Proposition 5 Within a global conduction band, the field inside the crystal can be represented by Bloch waves only.

\section{CONCLUSION}

We have shown that it is important to distinguish between various kinds of conduction bands: there may be non propagative modes that result from the local bifurcation of a propagative mode or all the non propagative modes may be made out of global bifurcation of propagative modes. In the first case, an important part of the field inside the structure is made of non-propagative modes, but in the second case, the field writes in terms of Bloch waves only. Especially near a band edge, one should be very careful before deriving the behavior of the field solely by looking at the dispersion diagram: it does not take into account the evanescent waves. These results might be useful in studying beam propagation and superprism effects in photonic crystals [24] and also the phenomenon of spontaneous emission in a finite dielectric structure, for in that case the density of modes for photons derived without precaution from Bloch theory is certainly false because the atom modes can couple to non-propagating radiation modes [25]. 


\section{REFERENCES}

[1] http://home.earthlink.net/ jpdowling/pbgbib.htm

[2] E. Centeno, D. Felbacq, J. Opt. soc. Am. A 17 (2000) 320.

[3] J. P. Dowling, C. M. Bowden, J. Mod. Opt. 41 (1994) 435.

[4] H. Kosaka et al., Phys. Rev. B 58 (1998) 10096.

[5] B. Gralak, G. Tayeb and S. Enoch, J. Opt. Soc. Am. A 17 (2000) 6.

[6] S. Enoch, G. Tayeb and D. Maystre, Opt. Comm. 161 (1999) 171.

[7] D. Felbacq, B. Guizal and F. Zolla, J. Opt. A: Pure Appl. Opt. 2 (2000) L30 .

[8] M. Notomi, Phys. Rev. B 62 (2000) 10696.

[9] T. Minami, H. Ajiki, K. Cho, Physica E 13 (2002) 432.

[10] J. B. Pendry, J. Mod. Opt. 41 (1994) 209.

[11] R. Petit ed., Electromagnetic theory of gratings, Springer-Verlag, Berlin, 1980.

[12] A. J. Ward, J. B. Pendry, W. J. Stewart, J. Phys.: Condens. Matter 7 (1995) 2217.

[13] G. Granet, J. Chandezon, O. Coudert, J. Opt. Soc. Am. A 14 (1997) 1576.

[14] L. Li, J. Opt. Soc. Am. A 13 (1996) 1024.

[15] D. Felbacq, in preparation.

[16] J. Lekner, J. Opt. Soc. Amer. A 11 (1994) 2892

[17] D. Felbacq, B. Guizal, F. Zolla, Opt. Comm. 152 (1998) 119.

[18] V. Jikov, S. Kozlov, O. Oleinik, Homogenization of differential operators and integral functionals, Springer-Verlag, Berlin, 1994.

[19] D. Felbacq, G. Bouchitté, Waves in Random Media 7 (1997) 245.

[20] T. Kato, Perturbation theory for linear operators, Springer-Verlag, Tokyo, 1984.

[21] Th. Frankel, The geometry of physics, Cambridge University Press, 1997.

[22] D. Felbacq, B. Guizal, F. Zolla, J. Math. Phys. 39 (1998) 4604.

[23] D. Felbacq, J. Phys. A: Math. Gen. 33 (2000) 7137.

[24] R. Smaâli, D. Felbacq, in preparation.

[25] A. Moroz, Europhys. Lett. 46 (1999) 419. 


\section{Figures captions}

Figure 1: Sketch of the photonic crystal.

Figure 2: (a) Absolute value of the eigenvalues of $\mathbf{T}_{\theta}(\lambda)$ for $\varepsilon_{1}=9, \varepsilon_{2}=\varepsilon_{\text {ext }}=1, h / d=$ $1, h_{1} / d=0.68, d_{1} / d=0.35, s$-polarized waves.

(b) Real part of the spectrum of $\mathbf{T}_{\theta}(\lambda)$. The arrows indicate a local and a global bifurcation.

(b) Dispersion diagram $(\beta, \lambda / d)$ for the parameters of figure 2 (a).

Figure 3: Evolution of the gaps with $\theta$. The white regions correspond to band gaps.

Figure 4: Branching ratios for $\varepsilon_{e x t}=2.26, \varepsilon_{2}=4, \varepsilon_{1}=1, h / d=1, d_{1} / d=0.5, s$-polarized waves. $\left(\pi_{a}\right)$.

$$
\text { propagating ratio }\left(\pi_{p}\right), \triangle \text { : evanescent ratio }\left(\pi_{e}\right), * \text { : anti-evanescent ratio }
$$

Figure 5: Spectrum and branching ratios for the parameters of figure 4.

(a) $N=2$ (b) $N=4$ (c) $N=6$.

Figure 6: Reflected energy and its enveloppe given by (6), with $N=20$.

(a) $\varepsilon_{1}=9, \varepsilon_{2}=\varepsilon_{\text {ext }}=1, h / d=1, h_{1} / d=0.68, d_{1} / d=0.35, s$-polarized waves.

(b) $\varepsilon_{\text {ext }}=2.26, \varepsilon_{2}=4, \varepsilon_{1}=1, h / d=1, d_{1} / d=0.5, s$-polarized waves. 


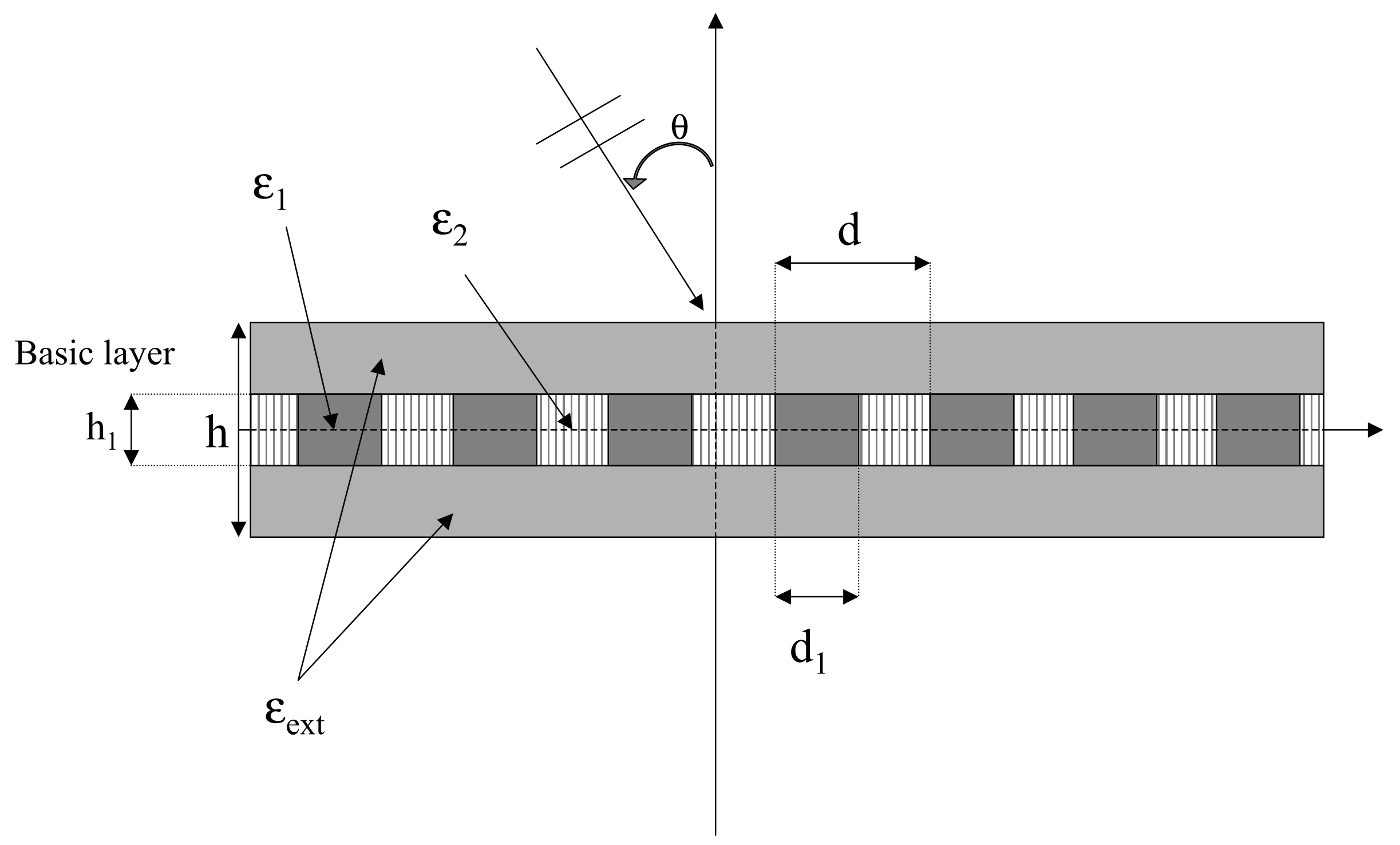




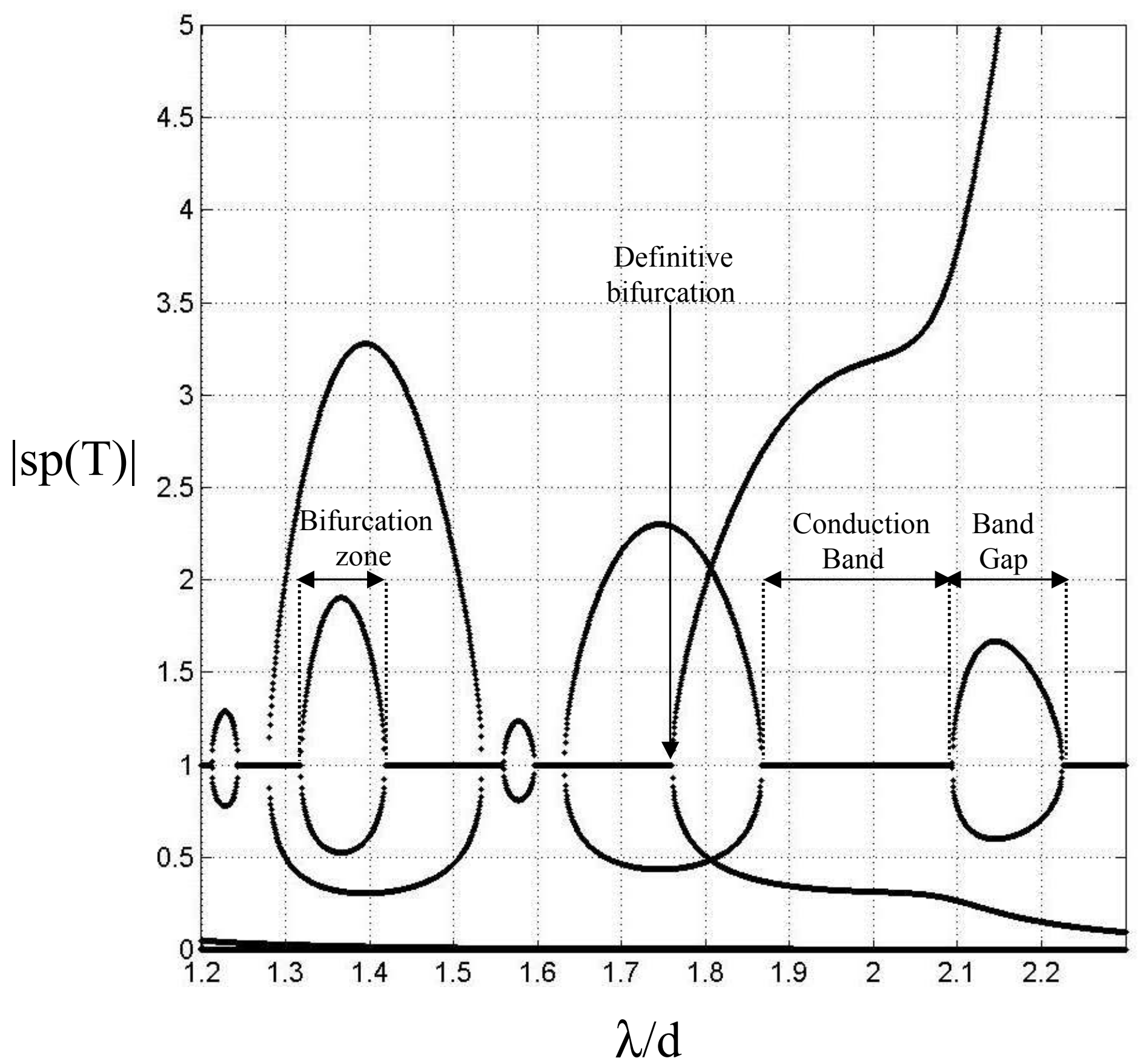




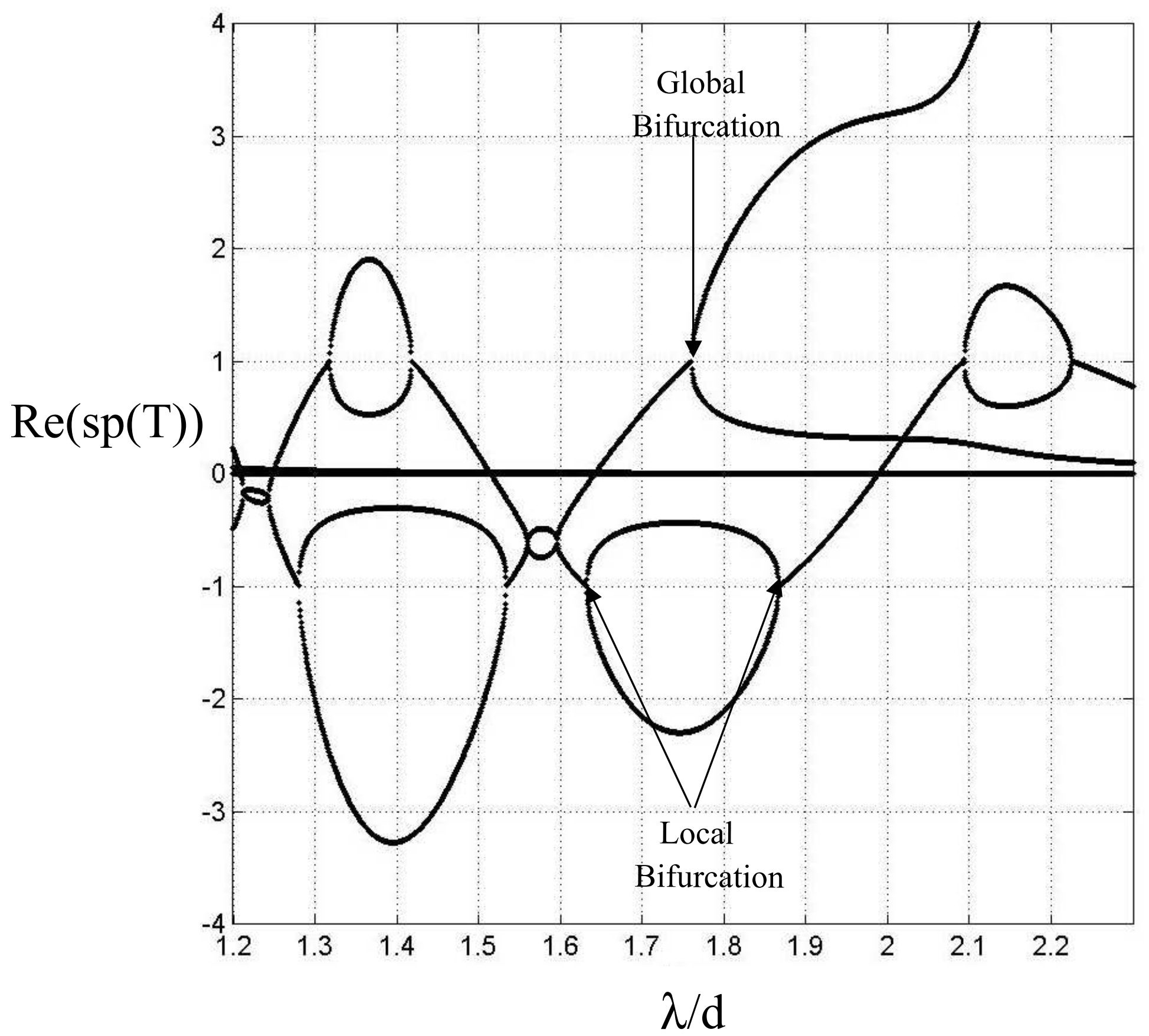




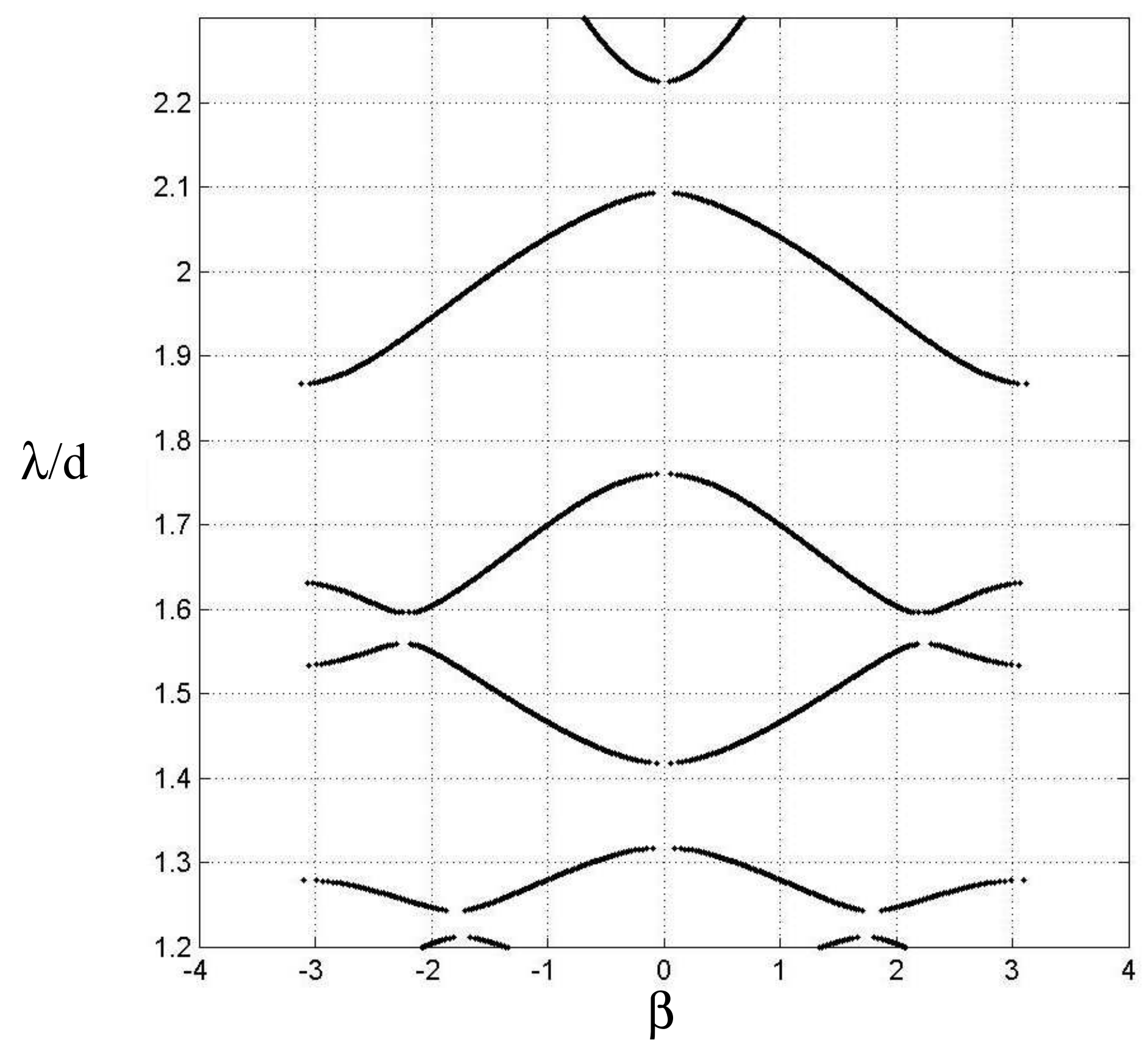




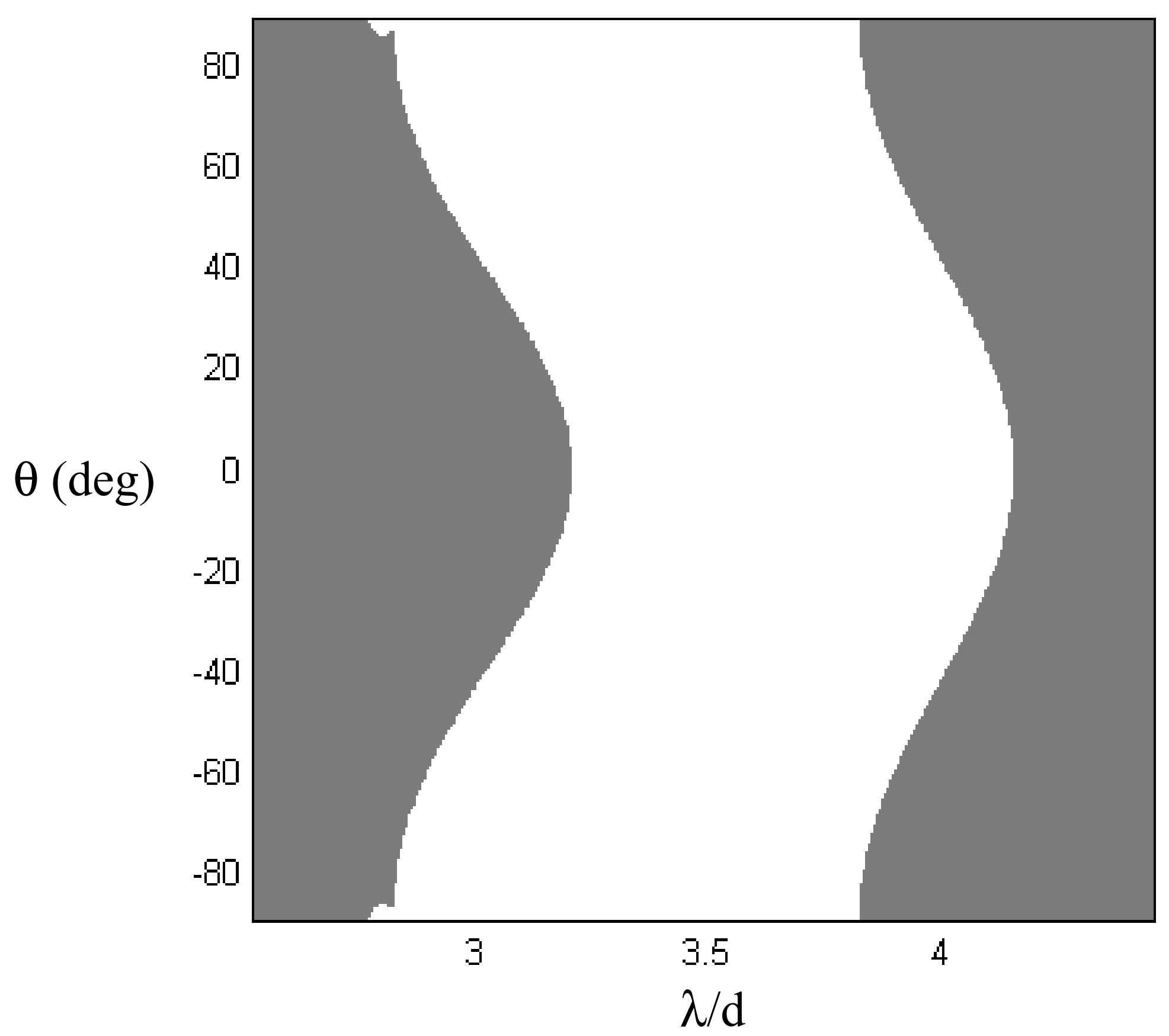




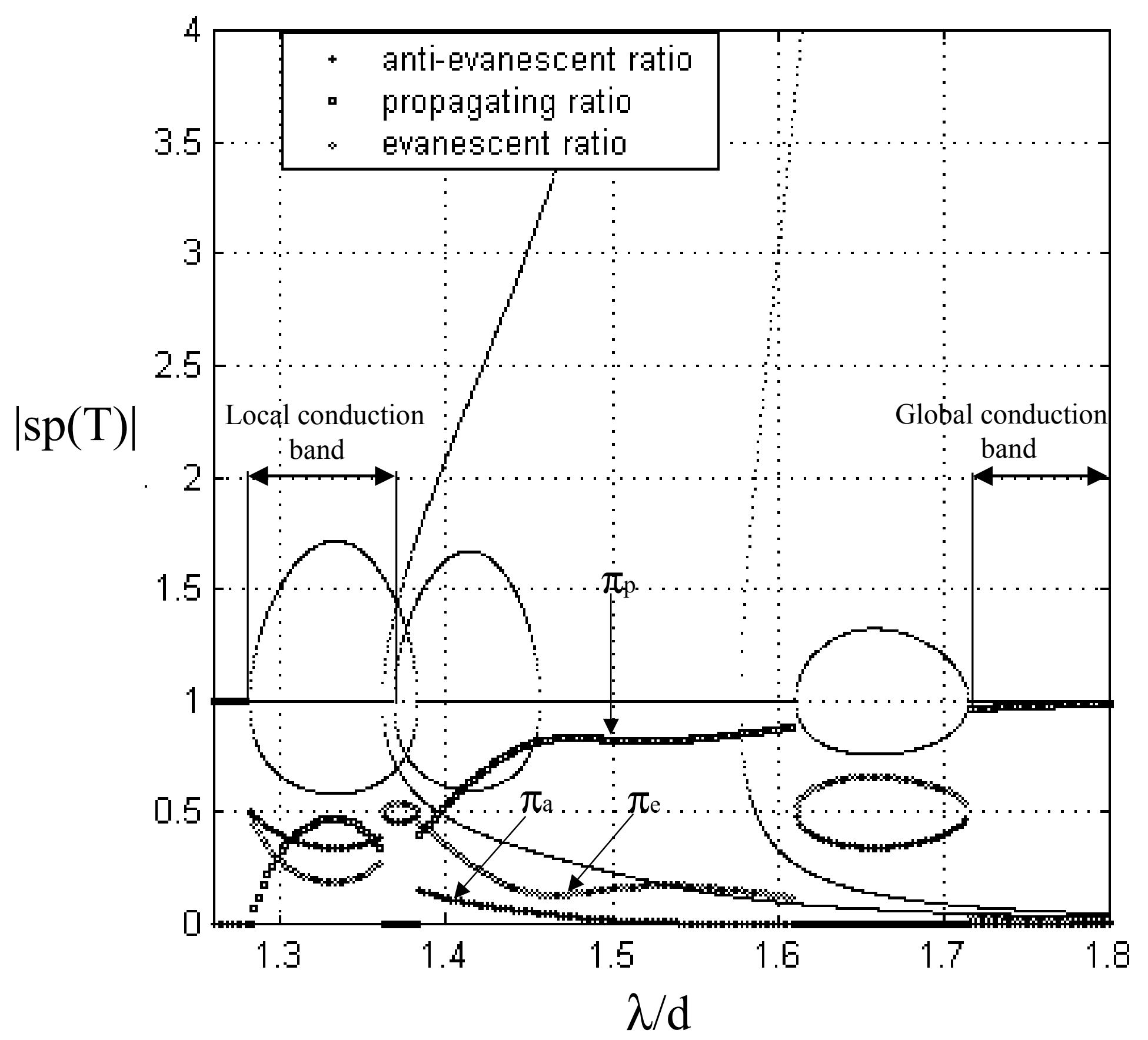




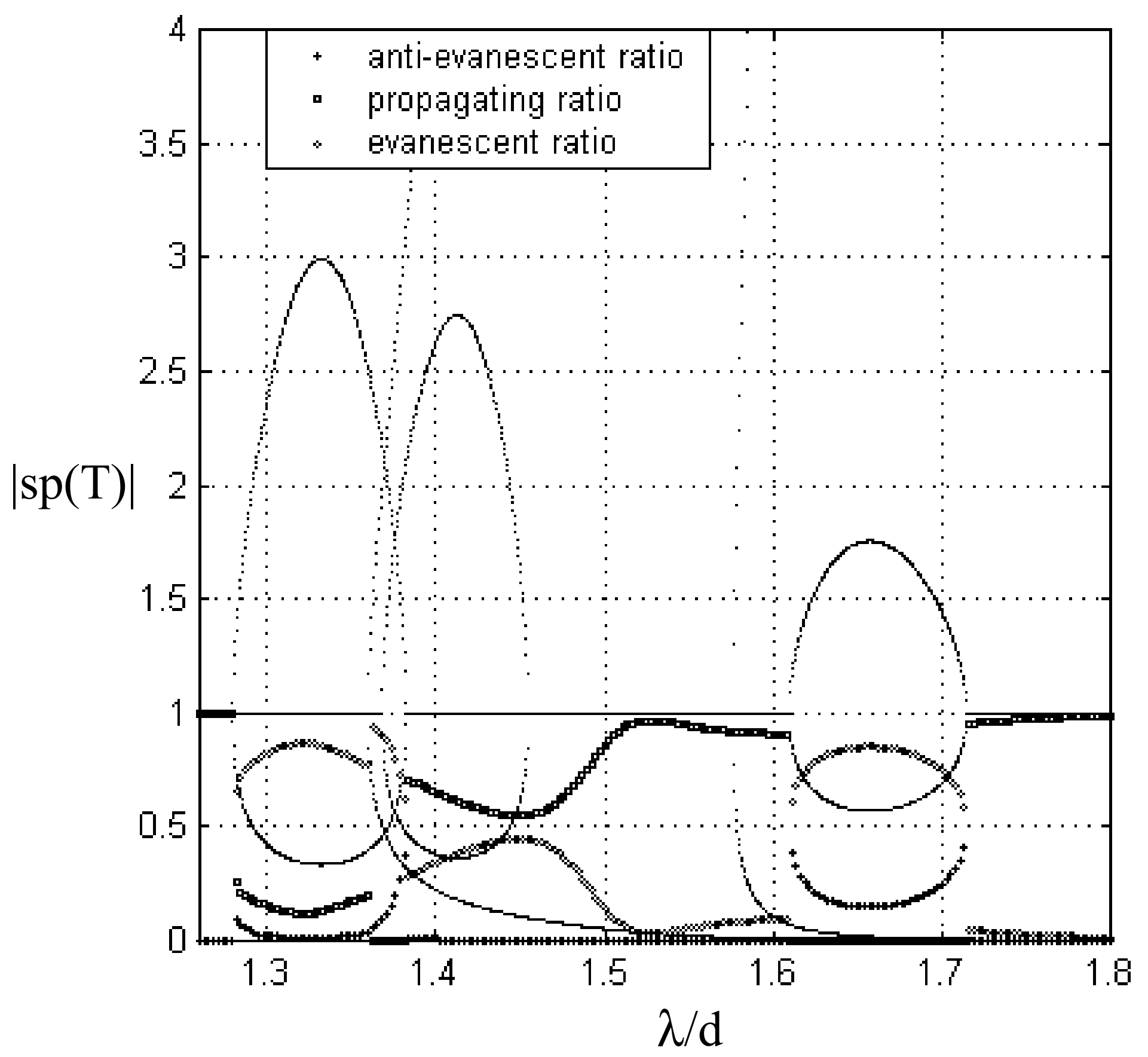




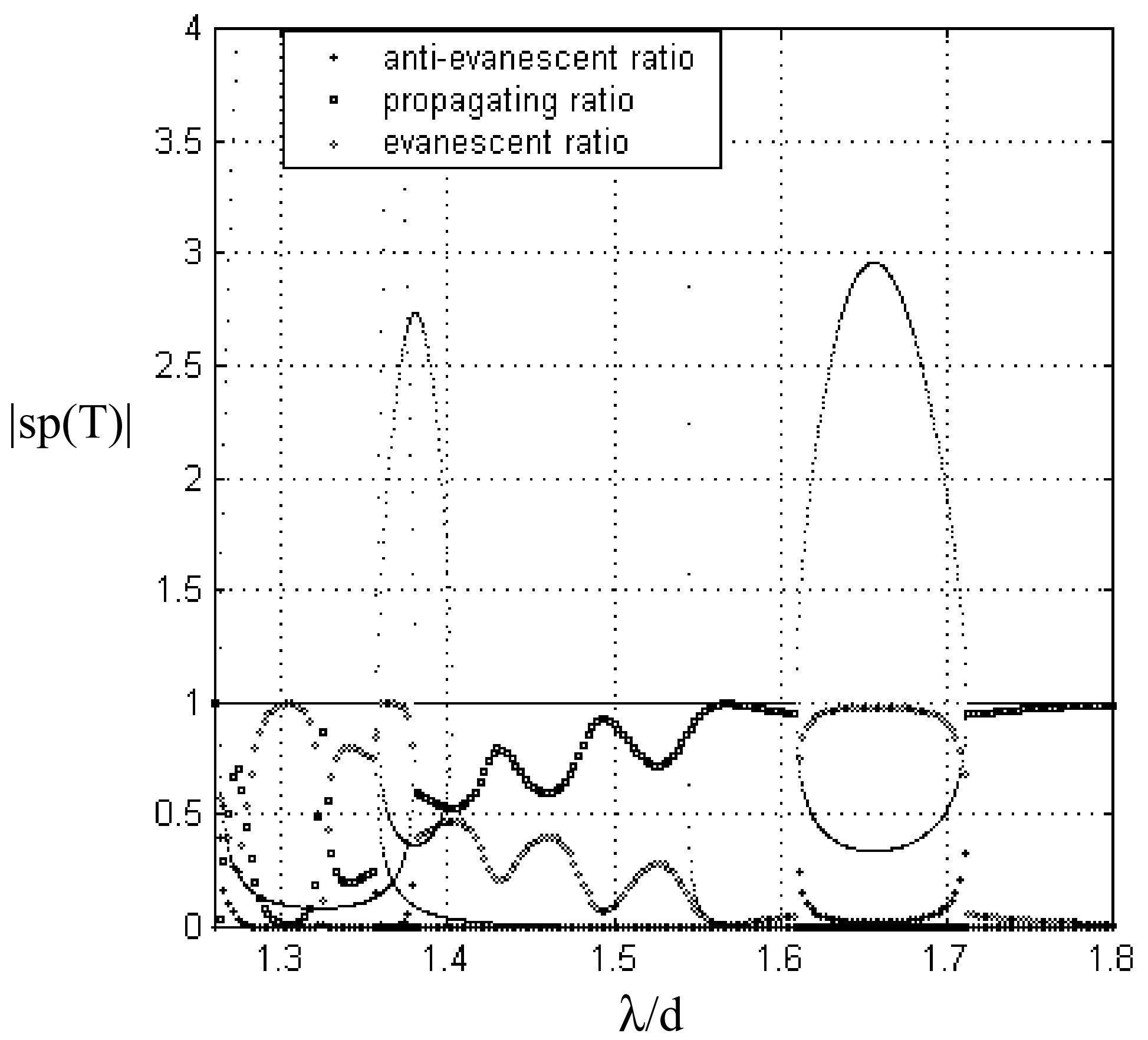




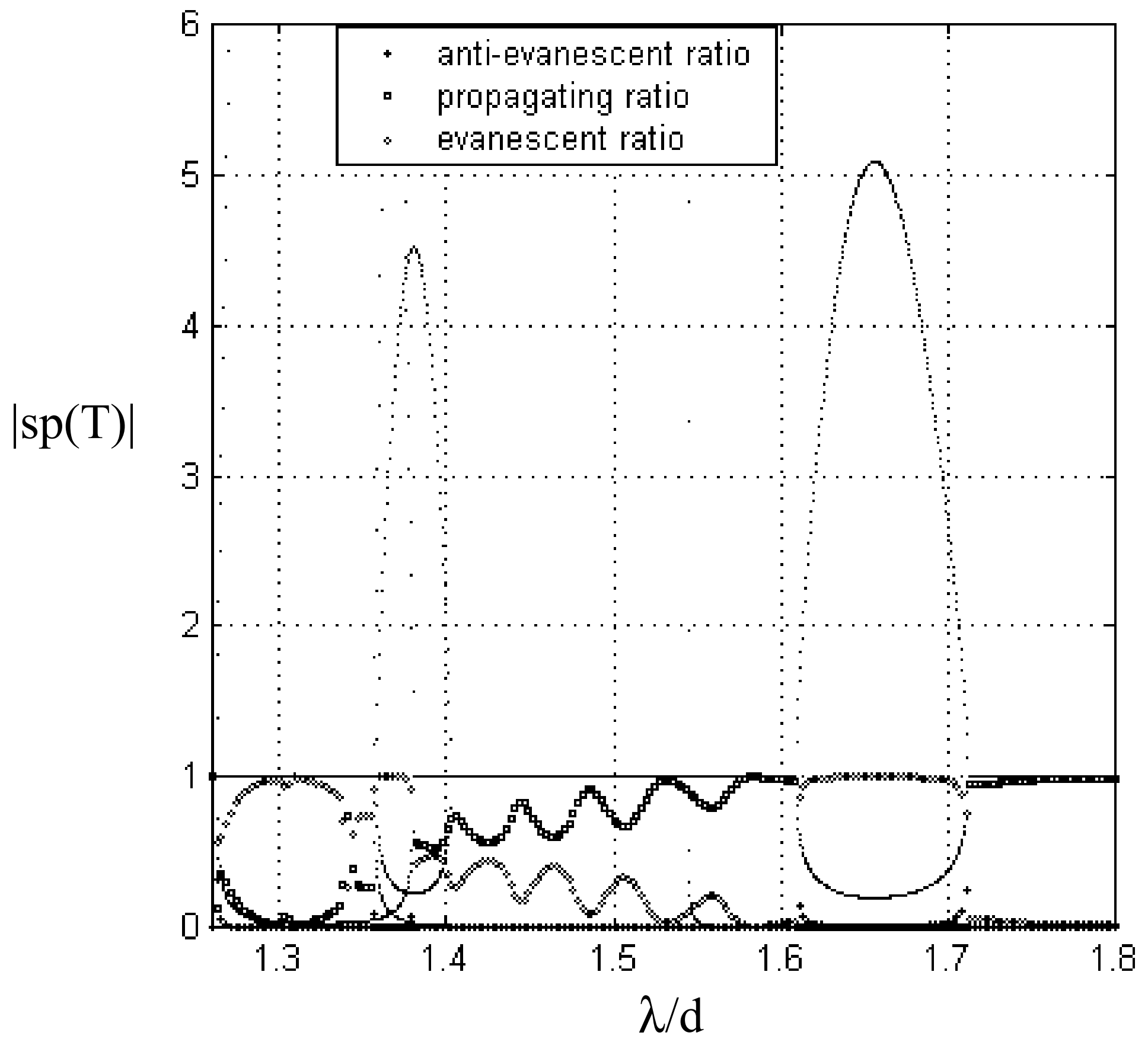




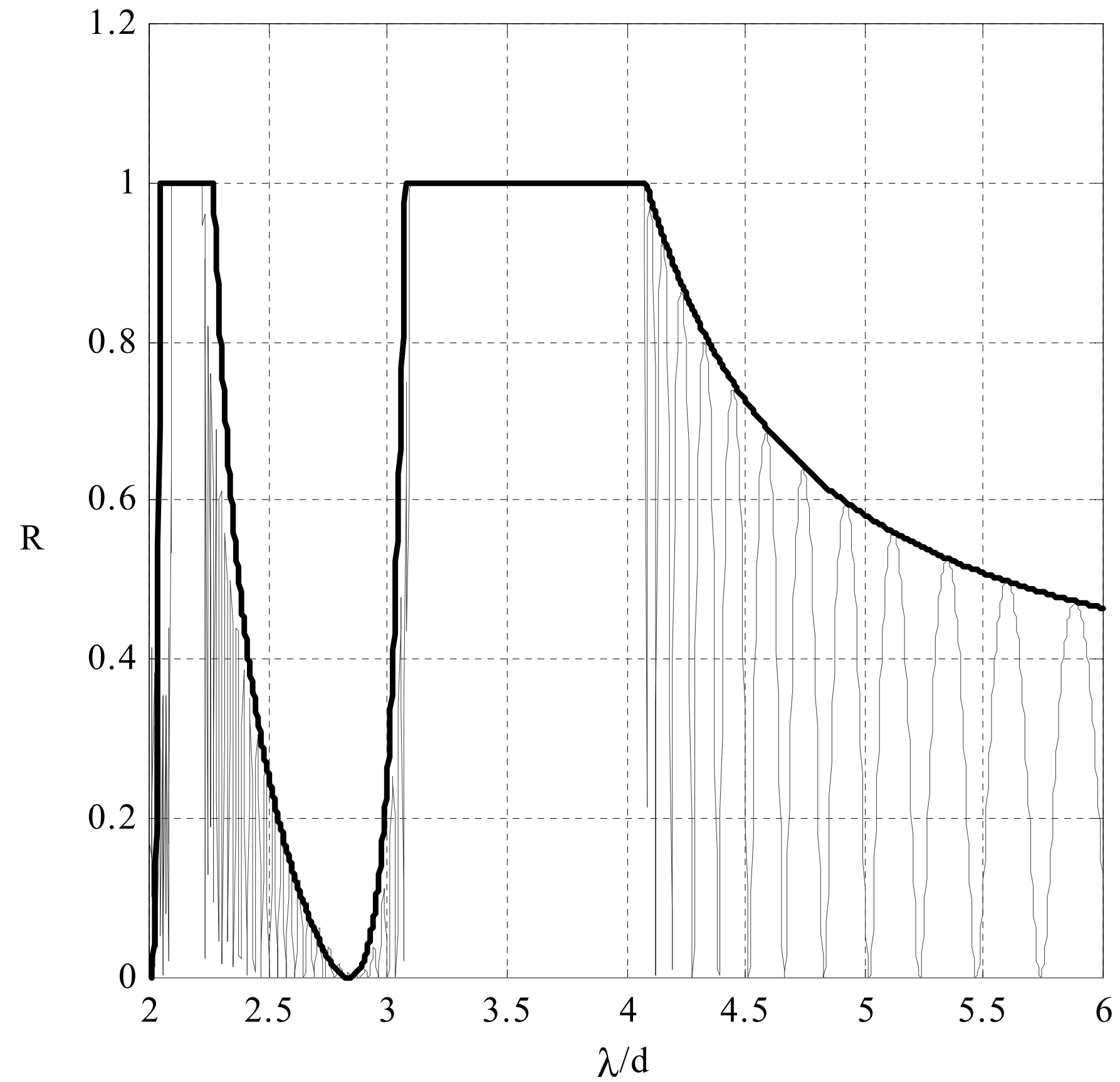




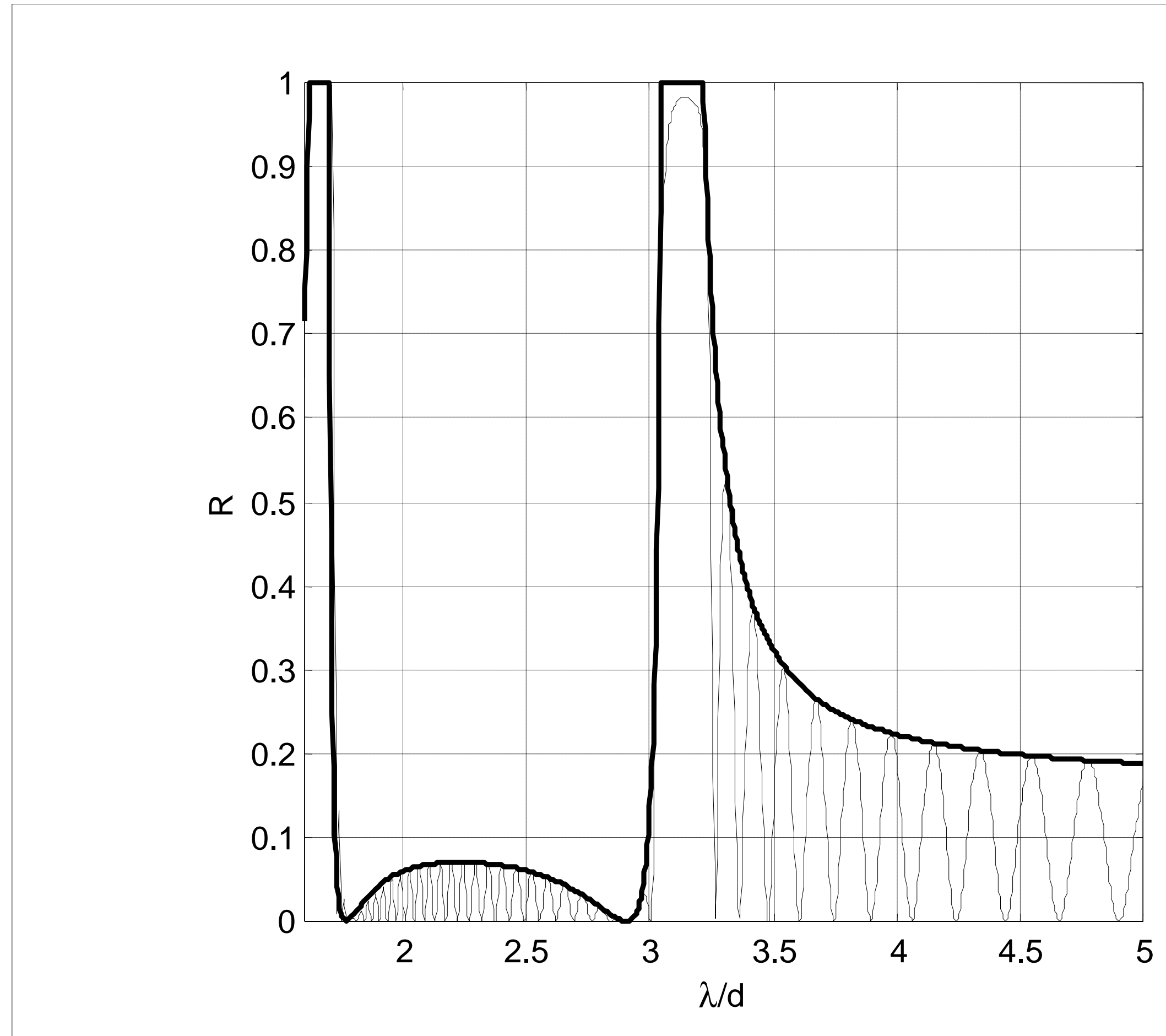

